\title{
Problèmes de Structures Universitaires
}

\author{
Marcel de Grandpré *
}

L'université québécoise du proche avenir. Textes choisis et présentés par René Hurtubise, Montréal, Hurtubise HMH, 1973, 403 p., Collection L'Homme dans la société, sous la direction de Guy Rocher et Pierre W. Bélanger.

Cet ouvrage contient deux parties. Il reproduit, en premier lieu, le rapport du Comité d'étude du Conseil des universités du Québec sur les objectifs de l'enseignement supérieur, soumis le 7 décembre 1972 (pages 21-146). L'autre partie comprend douze études dont dix ont été effectuées à la demande et aux frais du Conseil des universités dans le but d'alimenter la réflexion des auteurs du rapport (pages 146-403).

La présentation de l'ouvrage (pages 11-19) est de René Hurtubise, directeur général de la Conférence des recteurs et principaux des universités du Québec. L'université québécoise, souligne-t-il, se situe dans une société qui, dans le proche avenir, oscillera, sans doute, entre les caractéristiques d'une société postindustrielle, risquant de sacrifier la personne à l'efficacité scientifique et technique, et celles d'une société centrée sur la personne. L'université devra participer à ce choix, apporter les lumières de la science et de l'expérience de tous les pays. Elle le fera à l'intérieur du système social et du système d'enseignement, mais son rôle sera d'y cultiver les dynamismes de renouvellement. En jouant ce rôle, l'université s'adaptera elle-même de façon constante. Elle devra modifier ses structures administratives et pédagogiques pour que l'on puisse y acquérir le savoir tout au long de la vie (formation continue), y revenir quand on le peut ou qu'on en sent le besoin (formation récurrente), se mettre au courant des dernières acquisitions du savoir sur les divers éléments d'un problème (interdisciplinarité) et, par-dessus tout, adapter ses méthodes didactiques à une population étudiante qui a besoin d'apprendre à apprendre, d'apprendre à se former elle-même (auto-éducation) pour devenir un ferment dynamique dans la société (créativité).

C'est là le cadre de pensée adopté par les auteurs du rapport du Comité des objectifs de l'enseignement supérieur. René Hurtubise présidait ce comité dont les autres membres étaient Lorenzo Roy, Pierre W. Bélanger et André Turmel. René Hurtubise a tésumé, chapitre par chapitre, ce rapport dans Stod, vol. III, No 1, 1973, p. 93-95.

* Marcel de Grandpré, directeur de la Section d'Éducation comparée et des Fondements de l'éducation, Faculté des sciences de l'éducation, Université de Montréal. 
On y traite les thèmes suivants: 1 . les universités dans un monde en changement. 2. l'éducation permanente, facteur intégrateur de toute politique d'éducation, 3. l'enseignement collégial et le premier cycle universitaire, 4. les études avancées et la recherche, 5. le milieu universitaire québécois, 6. l'organisation du système universitaire québécois.

Les considérations principales du rapport sont les suivantes. Un sous-développement relatif, aux plans économique, social et culturel, crée au Québec une situation d'urgence. La définition des objectifs est au cœur du malaise universitaire. Le principal objectif des universités, durant la prochaine décennie, sera d'apporter une contribution significative et qualitative au développement économique, social et culturel du Québec, étant entendu que le but ultime du développement est la qualité de la vie. L'éducation permanente devient le facteur intégrateur de toute politique d'éducation. Les principales règles du jeu sont : accessibilité universelle et égalité d'éducabilité ; ouverture, souplesse et responsabilité devant les changements de la demande ; pluralisme et diversité ; rationalisation dans l'utilisation des ressources; diversité des approches pédagogiques et des modes d'apprentissage; enfin, dans le contexte d'une véritable éducation continue, réflexion en profondeur et remise en cause de la structure et de la durée des études, réorientation des programmes dans la perspective d'un premier cycle polyvalent basé sur des prémisses spécifiées ; création d'un centre de synthèse interdisciplinaire qui, à travers la poursuite de recherches scientifiques, découvre peu à peu et développe les fondements communs aux diverses disciplines, ne cherchant qu'ensuite à en dégager les applications pour l'enseignement. Bref, le rapport propose des objectifs globaux, bipolaires, en tension et, par conséquent, toujours dynamiques.

Il est difficile de dégager l'essentiel d'un document de ce genre. On pourrait estimer que le thème fondamental est l'organisation d'un système universitaire. Les universités du Québec ne peuvent plus être et ne sont plus des institutions isolées, indépendantes. Ensemble elles constituent un réseau, un système. On doit y assurer un équilibre dynamique entre centralisation et autonomie. La responsabilité devrait en appartenir au Conseil des universités, doté pour cela des pouvoirs nécessaires.

Pour les études, la suggestion la plus originale, ou du moins la plus frappante, voudrait que l'on répartisse les études supérieures en trois cycles de deux ans. Le but principal est de faire obtenir le premier grade après deux ans.

Le nom même du Comité des objectifs de l'enseignement supérieur nous indique l'orientation du rapport. Les rédacteurs ont, en effet, voulu faire le point, surtout dans les deux premiers chapitres, et bien situer les problèmes de l'enseignement supérieur au Québec dans l'ensemble des idées que l'on soulève aujourd'hui à propos des universités. Ils ont réussi à en dresser une synthèse à la fois valable et intéressante, bien que sommaire.

Le livre contient, en outre, douze études rédigées pour le Comité par les auteurs suivants : André Barsony, Gaëtan Daoust, Léon Dion, Fernand Dumont, Henri Favre, 
Nicole Gagnon, André Grou, Michel Leclerc, Ygal Leibu, Guy Rocher, Paul-Martel Roy et André Turmel, tous engagés dans l'enseignement, la recherche ou l'administration universitaire. Comme nous l'avons mentionné plus haut, ces études couvrent à peu près le même terrain que le rapport. Elles ont fourni la matière sur laquelle a travaillé le comité. mentaires.

Cette présentation, dans les termes mêmes de l'ouvrage, appelle quelques com-

1. La publication de cet ouvrage peut rendre de grands services dans les universités et partout où l'on s'intéresse à l'enseignement supérieur. Il est, en effet, très difficile de se tenir au courant de toutes les idées qui se débattent, ainsi que des méthodes de recherche que l'on applique aujourd'hui à l'étude des systèmes d'enseignement. Les travaux préparés pour le Comité des objectifs en font un inventaire assez complet. Des spécialistes mettent à notre portée des ouvrages parfois hautement techniques et font la synthèse de débats souvent complexes.

2. Le Comité des objectifs s'est inspiré de la façon de procéder des documents analogues produits au cours des dernières années. Il condense de manière systématique le contenu de la recherche tel qu'exposé par les douze études. Dans chaque secteur, il dégage un «modèle » qui sert ensuite à évaluer ce qui existe, à imaginer une prospective, une «utopie», et à choisir des objectifs conformes au modèle adopté. On compte, par cette méthode, faire évoluer l'université en mettant à contribution l'analyse scientifique la plus à la page.

3. Cette dialectique comporte des caractères propres dont il faut prendre conscience. Autrement, il serait difficile d'en tirer profit aussi bien que d'en saisir les limites. Les données résumées dans le rapport sont très nombreuses et l'on nous renvoie scrupuleusement aux sources. Réduites aux dimensions de modèles maniables, ces données prennent la forme d'un répertoire d'énoncés très brefs que l'on admet en les supposant prouvés ailleurs. Ces énoncés deviennent ainsi des postulats. Bien agencés, ils entraînent aisément l'adhésion de l'esprit et, d'étape en étape, ils conduisent à accepter les applications qu'on en tire comme des conséquences rigoureuses de la recherche scientifique. On doit donc avoir bien clairement à l'esprit que toute la charpente repose sur des posutulats et tient d'eux toute leur valeur.

4. Ces postulats se présentent d'ailleurs sous forme d'énoncés dogmatiques, comme les «règles du jeu» des pages 52 à 63 : «accessibilité universelle à l'éducation et égalité devant l'éducation », etc. Or, à maintes reprises, au cours de l'ouvrage, on nous signale des postulats qu'il fallait admettre il y a dix ans et qui sont déjà caducs. Ainsi, le célèbre «qui s'instruit s'enrichit». Une lecture éclairée de l'ouvrage suppose donc, en bonne méthodologie, qu'on identifie au passage chaque postulat, qu'on évalue son degré de certitude et qu'on suive tout le long du raisonnement la bande d'improbabilité qui en découle. 
5. La méthode a servi à rédiger le Rapport Parent, introduisant un « humanisme sociologique » et «une optique fondée sur la rationalité et le respect de l'individu» (p. 33). On a déjà signalé que cette méthode est désincarnée. Les modèles sont abstraits, tirés d'autres milieux. L'analyse du milieu québécois est abstraite également. Il est paradoxal que l'on ramène l'observation du milieu à des énoncés «transculturels 》 en ce sens qu'on élimine les données concrètes, historiques et culturelles, pour dégager des schémas communs à toute autre société. Le paradoxe surprend davantage en période de prise de conscience de soi de la société québécoise. Il pourrait en découler une «acculturation» au milieu nord-américain, une assimilation culturelle dont les conséquences devraient entrer tout de suite dans notre prospective. La plupart des problèmes qu'a entraînés l'application du Rapport Parent, aux niveaux primaire, secondaire et collégial, n'étaient-ils pas clairement inclus dans l'adoption de modèles nord-américains, adaptés sans doute à leurs milieux sociaux, mais moins adéquats ici, dans la mesure où existe une société québécoise dotée d'une personnalité ?

6. Le Rapport du Comité des objectifs tire comme conséquence d'une société centrée sur la personne et du rôle intégrateur de l'éducation permanente, la nécessité d'une formation générale. Il déplore que le CEGEP ne soit pas la dernière étape de la formation générale, comme le voulait le Rapport Parent, mais, de fait, le début déjà très spécialisé de la formation professionnelle. Cela aussi n'était-il pas clair dans l'adoption d'une structure étroitement calquée sur les systèmes nord-américains ?

7. Nous pourrions proposer une technique différente. Dans les systèmes d'enseignement, on doit distinguer le contenu (les programmes d'études), l'institution (par exemple, la structure administrative de l'université) et l'autorité «politique 》 (ici, le Conseil des universités, la DGES, le ministère de l'Éducation, etc.). Une règle méthodologique valable pourrait être qu'il s'agit de trois structures connexes, mais nettement distinctes, et que l'on ne doit jamais lier la réforme d'une structure à celle de l'une des deux autres. Notre réforme scolaire récente tient de ce type d'erreur une bonne part de ses échecs.

8. Ainsi, l'enseignement de culture générale préparant à faire de bonnes études supérieures a paru lié au «cours classique » traditionnel au Québec et aux collèges classiques. La redistribution du pouvoir qui s'est faite pendant la «révolution tranquille »a entraîné d'un seul mouvement à la fois le cours classique et les collèges classiques. Ces derniers ne s'étaient pas assez rendu compte que le cours classique avait sa valeur propre, indépendante de l'institution. Incapables de formuler la théorie de leur enseignement, les collèges, en disparaissant comme institutions, ont entraîné la disparition du type de formation qu'ils n'étaient plus tout à fait seuls à donner.

9. Les collèges classiques, ou du moins nos réformateurs, auraient dû voir et dégager ces valeurs. Le cours classique contenait le principe d'uné formation générale basée sur des disciplines multiples. Nous avions là une méthode avec laquelle nous aurions pu et dû aborder les problèmes de l'éducation dans une société postindustrielle. La 
culture par les lettres, les arts, l'histoire, la philosophie reposait sur une tradition pédagogique de valeur mais insuffisamment formulée. La culture par les sciences y était encore tâtonnante mais elle comptait déjà un début de tradition. Le monde contemporain n'a pas su encore dégager de la technologie une culture générale. C'est le « défi 》 que posait au Québec sa réforme scolaire. Ce défi était original parce que le Québec était au confluent de la tradition classique et de la société postindustrielle.

10. Une notion absente du Rapport du Comité des objectifs, comme de nos considérations habituelles, est celle d'équipement collectif progressif. La méthode d'analyse à la mode part de généralisations récentes formulées de façon abstraite. Elle compare la réalité à ce «modèle ». Par l'analyse critique elle suscite ensuite dans le réel des antagonismes entre ce qui est conforme au modèle et ce qui en diffère. Elle prend alors appui sur cette dynamique pour transformer le milieu analysé. La conception ancienne du modèle était, au contraire, concrète. On prenait pour «modèle » une personne ou une institution dont on estimait valables certains traits plus ou moins nombreux et l'on essayait de se les assimiler graduellement. La méthode était aussi dynamique, aussi capable d'introduire des transformations. Mais elle partait de la réalité concrète qu'elle essayait d'améliorer. Pour une collectivité, comme la société québécoise, la perspective est très différente. Le modèle sociologique fait abstraction des caractères propres à l'institution, au milieu social particulier. L'autre méthode faisait prendre conscience du caractère graduel du progrès, de l'accumulation des apports de chaque individu et de chaque génération. Elle donnait le sens de la continuité. Elle apprenait la modestie. Elle était une culture.

11. L'objectif central de l'ouvrage pourrait fort bien se ramener à la formulation suivante : «L'éducation tendant vers la permanence serait le processus organique intégré, conçu et réalisé de manière à fournir à la personne, outre des connaissances, les méthodes d'en découvrir de nouvelles au gré des besoins et des intérêts, de s'informer, de formuler les problèmes et de les résoudre, d'opérer des choix et d'agir non seulement dans le sens de ses valeurs propres mais aussi comme facteur de changement social » (p. 334). On ne saurait mieux exprimer l'idéal de "l'honnête homme » issu de la Renaissance. C'est celuici qui a découvert et fait l'Amérique du Nord. Il n'est peut-être pas trop tard pour redécouvrir dans ce continent ce type d'efficacité.

12. Pour conclure ces réflexions que suggère l'ouvrage du Comité des objectifs, on pourrait souligner le mérite des auteurs. Ils ont condensé une somme de données extrêmement riche. Ils l'ont mise à la portée du milieu universitaire. $\bar{A}$ une étape ultérieure, des études semblables pourront sans doute, souhaitons-le, compter sur la collaboration de disciplines plus variées. Davantage interdisciplinaires, elles emprunteront un langage moins technique et des préocccupations plus complètes. En attendant, il faut féliciter et remercier les «technocrates » qui ont fait un tel effort pour nous faire béné. ficier de leurs recherches et de leurs réflexions. 